\title{
Transgenerational effects of the endocrine disruptor vinclozolin on the methylation pattern of imprinted genes in the mouse sperm
}

\author{
Christelle Stouder ${ }^{1}$ and Ariane Paoloni-Giacobino ${ }^{1,2}$ \\ ${ }^{1}$ Department of Genetic Medicine and Development and ${ }^{2}$ Swiss Center for Applied Human Toxicology, \\ University of Geneva Medical School, CMU, 1 Michel-Servet, 1211 Geneva 4, Switzerland \\ Correspondence should be addressed to A Paoloni-Giacobino at Department of Genetic Medicine and Development, \\ University of Geneva Medical School,CMU; Email: ariane.giacobino@unige.ch
}

\begin{abstract}
Endocrine-disrupting chemicals (EDCs), among which is the antiandrogen vinclozolin (VCZ), have been reported to affect the male reproductive system. In this study, VCZ was administered to pregnant mice at the time of embryo sex determination, and its possible effects on the differentially methylated domains (DMDs) of two paternally (H19 and Gt/2) and three maternally (Peg1, Snrpn, and Peg3) imprinted genes were tested in the male offspring. The CpGs methylation status within the five gene DMDs was analyzed in the sperm, tail, liver, and skeletal muscle DNAs by pyrosequencing. In the sperm of controls, the percentages of methylated CpGs were close to the theoretical values of $\mathbf{1 0 0}$ and $\mathbf{0} \%$ in paternally or maternally imprinted genes respectively. VCZ decreased the percentages of methylated CpGs of $\mathrm{H19}$ and $\mathrm{Gt} / 2$ (respective values 83.1 and 91.5\%) and increased those of Peg1, Snrpn, and Peg3 (respective values 11.3, 18.3, and $\mathbf{1 1 . 2 \%}$ ). The effects of $\mathrm{VCZ}$ were transgenerational, but they disappeared gradually from F1 to F3. The mean sperm concentration of the VCZ-administered female offspring was only $56 \%$ of that of the controls in the F1 offspring, and it was back to normal values in the F2 and F3 offspring. In the somatic cells of controls, the percentages of methylated CpGs were close to the theoretical value of $50 \%$ and, surprisingly, VCZ altered the methylation of Peg3. We propose that the deleterious effects of VCZ on the male reproductive system are mediated by imprinting defects in the sperm. The reported effects of EDCs on human male spermatogenesis might be mediated by analogous imprinting alterations.

Reproduction (2010) 139 373-379
\end{abstract}

\section{Introduction}

Exposure to environmental pollutants may induce changes in gene expression not involving changes in the underlying DNA sequence, but involving the so-called epigenetic changes. Imprinting is an epigenetic form of gene regulation that mediates a parent-of-origin-dependent expression of the alleles of a number of genes. It occurs at specific sites within or surrounding the gene called differentially methylated domains (DMDs). Within a DMD, one parental allele is methylated on all or the majority of its $\mathrm{CpG}$ dinucleotides, and the opposite parental allele is methylated on none or a small percentage of its CpG dinucleotides. DNA methylation of imprinted gene DMDs results in the silencing of the methylated allele (Li et al. 1993, Kaneda et al. 2004). An epigenetic reprogramming initiated by the erasure of preexisting DNA methylation marks takes place during the embryonic development in primordial germ cells. Imprint reestablishment follows in both the female and the male germline during gametogenesis (Lee et al. 2002).
In the past century, a decline in sperm counts has been documented in young healthy men of industrialized countries, which could be responsible for the currently observed decline in fertility rates. The rapidity of the changes suggested that environmental factors may play a role (Andersson et al. 2008). Among them are the endocrine-disrupting chemicals (EDCs), which are substances in our environment and food that interfere with the biosynthesis, metabolism, and action of hormones. The EDCs stand for a broad class of molecules among which are the organochlorinated pesticides (Diamanti-Kandarakis et al. 2009). Several studies performed in humans reported that exposure to pesticides may decrease spermatogenesis and male fertility (Roeleveld \& Bretveld 2008).

It has recently been shown that imprinting defects are associated with decreased spermatogenesis. Indeed, partial methylation errors affecting the CpGs of paternally or maternally methylated genes (Roeleveld \& Bretveld 2008) and methylation defects in the maternally 
methylated H19 gene (Marques et al. 2010) were observed in the sperm of oligospermic or azoospermic patients respectively.

Studies in rodents strongly suggest a link between EDCs and the reproductive system. Vinclozolin (3-(3,5-dichlorophenyl)-5-methyl-5-vinyl-1,3-oxazolidine-2,4-dione, VCZ), a fungicide used to treat fruits and vegetables, was found to exert significant antiandrogenic effects. When administered to pregnant female mice during the gestational period of gonadal sex determination, VCZ was found to affect the offspring. It masculinized females (longer urethras), feminized males (hypospadias), and increased the expression of progesterone receptors in both genders (Buckley et al. 2006). It also induced decreases in the anogenital distance and in the prostate weight (Buckley et al. 2006, Elzeinova et al. 2008). Eventually, VCZ affected spermatogenesis inducing decreases in sperm number and motility, sperm head abnormalities, and apoptosis in the seminiferous tubules germ cells (Shimamura et al. 2002, Anway et al. 2005, 2006, 2008, Buckley et al. 2006, Elzeinova et al. 2008). An intriguing feature of VCZ is that the effects on male genital tract and spermatogenesis were found to be transgenerational, extending from F1 to F4 (Anway et al. 2005, 2006, 2008). An outcross of a VCZ generation female with a wild-type male had no phenotype, suggesting that the male germline is responsible for the transmission of the phenotype (Anway et al. 2006). Studies performed using the same protocol, i.e. VCZ administration to pregnant female rats during the gestational period of gonadal sex determination, failed to demonstrate any change in spermatogenesis. The reason for this discrepancy has not been identified (Schneider et al. 2008, Inawaka et al. 2009).

In the mouse studies in which a transgenerational effect of VCZ was observed, the possibility of an epigenetic effect of the endocrine disruptor on the male germline was investigated. A methylation-sensitive restriction enzyme study revealed altered methylation patterns on various DNA fragments, called 'imprintedlike gene/DNA sequences', in the F1-F3 offspring sperm (Anway et al. 2005). However, these studies were only preliminary, and it is still not known whether or not these DNA fragments are associated with genes exhibiting imprinted (i.e. monoallelic, parent-of-origin dependent) expression.

A few mouse and human DMDs have been well characterized. They are, in particular, the DMDs of the maternally expressed paternally methylated H19 (Tremblay et al. 1997) and Gt/2 (Li et al. 2004) genes and the paternally expressed maternally methylated small nuclear ribonucleoprotein polypeptide N (Snrpn) (Shemer et al. 1997), Peg1 (Kaneko-Ishino et al. 1995), Peg3 (Kuroiwa et al. 1996), and the potassium channel 1 (Kcnq1) (Smilinich et al. 1999) genes.

The aim of the present study was to evaluate, more systematically than ever before, the possible deleterious effects of VCZ administration in mice during pregnancy on imprinted genes. The study was performed by testing the possible methylation defects in the DMDs of two paternally (H19 and Gt/2) and three maternally (Peg1, Snrpn, and Peg3) imprinted genes in the sperm as well as in the tail, liver, and skeletal muscle DNAs of the male offspring over three generations.

\section{Results}

VCZ or vehicle only was administered to five pregnant female mice during the gestational period of gonadal sex determination, and the methylation status of the paternally methylated $\mathrm{H} 19$ and Gt/2 genes and that of the maternally methylated Peg1, Snrpn, and Peg3 genes were studied by DNA bisulfite treatment in male offspring over three generations (F1-F3). For each imprinted gene, a number of CpGs varying between 5 and 23 were analyzed. The DNA pyrosequencing technique allows to measure the amount of methylated CpGs as compared to total (methylated and nonmethylated) CpGs.

In the sperm of control male offspring, the numbers of methylated $\mathrm{CpGs}$ were close to the theoretical values of 100 and $0 \%$ of the total CpGs in paternally or maternally methylated genes respectively (Fig. 1). VCZ induced dramatic changes in the sperm of male offspring (F1). The number of methylated CpGs of $\mathrm{H} 19$ and Gt/2 were decreased (respective values 83.1 and $91.5 \%$ ), and those of Peg1, Snrpn, and Peg3 increased (respective values $11.3,18.3$, and $11.2 \%$ ). The VCZ effects were all highly significant $(P<0.001$; Fig. 1$)$. As demonstrated by the S.E.M., the inter-individual variations were relatively small. It is noteworthy that all the sperm methylated

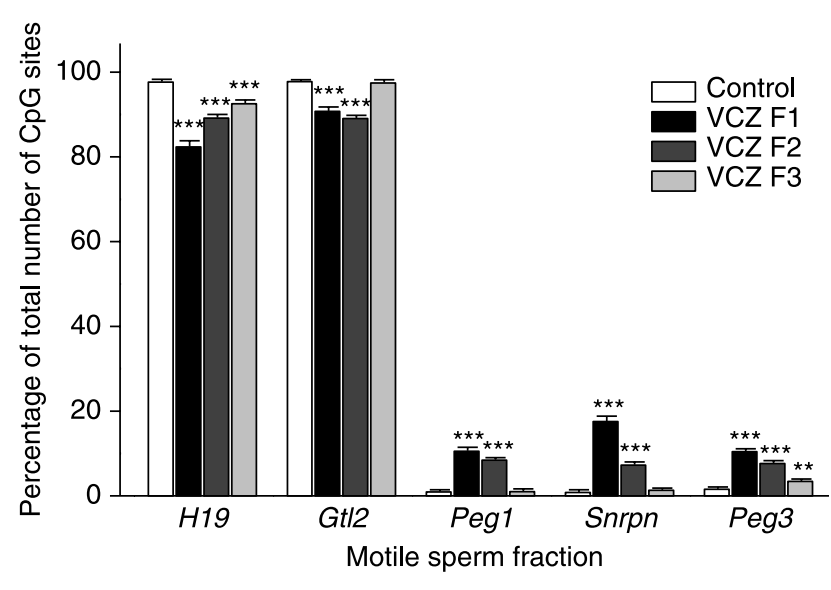

Figure 1 Methylation status of the paternally methylated $\mathrm{H} 19$ and Gt/2 genes and of the maternally methylated Peg1, Snrpn, and Peg3 genes in the motile sperm fraction of the F1-F3 offspring of control and of vinclozolin-administered female mice. Control: vehicle only.

VCZ: vinclozolin. The results are the means \pm s.E.M. of 8-15 mice. They represent the number of methylated $\mathrm{CpG}$ sites and are expressed as the percentage of the total number of CpG sites. ${ }^{* * *} P<0.001$ and ${ }^{* *} P<0.01$ versus respective controls. 
CpG values of the VCZ-administered female offspring differed from control values with no overlap in the sperm methylated $\mathrm{CpG}$ values between the two groups $(n=10$ and 15 respectively). The modifications of each gene methylation status were found to be evenly distributed between the $\mathrm{CpGs}$, with no particular one being more affected than the others. Therefore, no specific CpG sites were found to be more or less sensitive to VCZ than the overall sites within the region studied. The effects of VCZ were still present and highly significant $(P<0.001)$ in the F2 offspring sperm (Fig. 1). However, a trend toward a recovery between F1 and F2 sperm was observed, with the methylated $\mathrm{CpG}$ values being significantly higher in the F2 offspring than in the F1 offspring for H19 $(P<0.001)$ and lower in the F2 offspring than in the F1 offspring for Peg1, Snrpn, and Peg3 $(P<0.05, P<0.001$, and $P<0.001$ respectively). Only Gt/2 was as much affected in the F2 offspring as in the F1 offspring. In the F3 offspring, the effects of VCZ could not be detected anymore in Gtl2, Peg1, and Snrpn, and they were significantly less marked in $\mathrm{H} 19$ and Peg3 $(P<0.01$ and $P<0.001$ respectively). An outcross of a VCZ generation F1 female with a wild-type male resulted in a methylation pattern of H19, Gt12, Peg1, Snrpn, and Peg3 in the sperm of the F2 offspring that did not differ from that of the control (not shown; Anway et al. 2006).

In the tail, which represents a heterogenous sample of somatic cells, the amount of methylated CpGs in control offspring was close to $50 \%$ of the total methylated+nonmethylated CpGs in all the imprinted genes tested. This value is in total agreement with the theoretical value expected for imprinted genes in which DNA methylation/unmethylation is present on one of the two parental alleles only (Fig. 2). A few differences were observed between control and VCZ-administered female offspring. In the F1 offspring, the methylated $\mathrm{CpG}$ values were significantly higher in H19 and lower in Snrpn than in the controls. In the F2 and F3 offspring, they were significantly lower in Peg3 than in the controls (Fig. 2).

To investigate further the finding that the VCZ-induced changes in the methylation patterns of imprinted genes are not restricted to male germline cells, the methylation patterns of our five target genes were analyzed in liver and skeletal muscle. As observed in the tail, the amount of methylated CpGs in the control offspring was close to the theoretical value of $50 \%$ of the total CpGs in all the imprinted genes tested (Figs 3 and 4). Again, a few differences were observed between control and VCZ-administered female offspring. In the liver of the F1 offspring, the methylated CpG values were significantly lower in Snrpn than in the controls. In the F2 offspring, they were significantly lower in Peg1 than in the controls, and in the F2 and F3 offspring, they were significantly lower in Peg3 than in the controls (Fig. 3). In the skeletal muscle of the F1 offspring, the methylated $\mathrm{CpG}$ values were significantly lower in Snrpn than in the

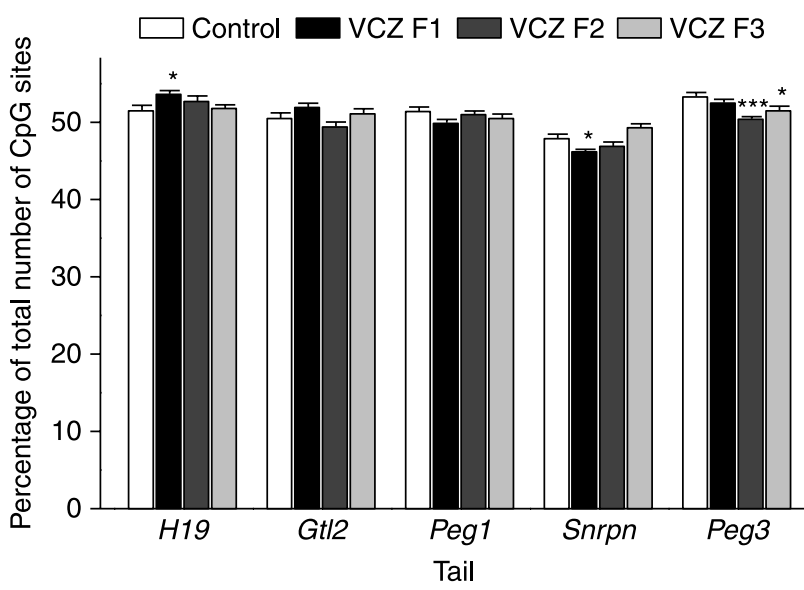

Figure 2 Methylation status of the paternally methylated $\mathrm{H} 19$ and $\mathrm{Gt} / 2$ genes and of the maternally methylated Peg1, Snrpn, and Peg3 genes in the tail of the F1-F3 offspring of control and vinclozolin-administered female mice. The results are the means \pm s.E.M. of 8-15 mice.

They represent the number of methylated $\mathrm{CpG}$ sites and are expressed as the percentage of the total number of $\mathrm{CpG}$ sites. ${ }^{* * *} P<0.005$ and $* P<0.05$ versus respective controls.

controls. In the F2 offspring, they were significantly lower in Snrpn and Peg3 than in the controls (Fig. 4). It is noteworthy that the changes induced by VCZ in the somatic cells are not as erratic as they look, with them being reproducibly observed for the F1 offspring Snrpn in the tail, liver, and muscle, and for the F2 offspring Peg3 in the tail, liver, and muscle.

The possibility that the observed changes in the methylation pattern of imprinted genes in the sperm might have biological consequence was tested. A sperm count was performed in five controls and in six to eight

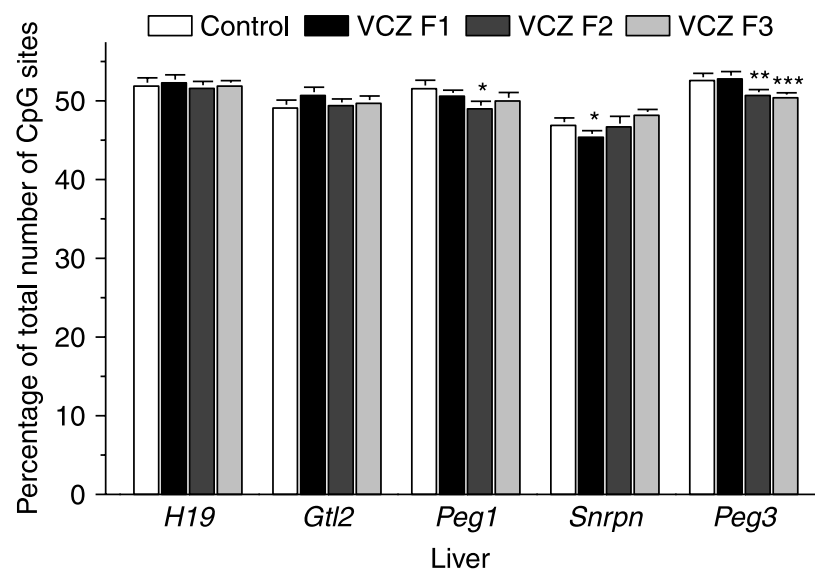

Figure 3 Methylation status of the paternally methylated $\mathrm{H} 19$ and $\mathrm{Gt} / 2$ genes and of the maternally methylated Peg1, Snrpn, and Peg3 genes in the liver of the F1-F3 offspring of control and vinclozolin-administered female mice. The results are the means \pm s.E.M. of 8-15 mice.

They represent the number of methylated $\mathrm{CpG}$ sites and are expressed as the percentage of the total number of $\mathrm{CpG}$ sites. ${ }^{* * *} P<0.001$; ${ }^{* *} P<0.01$, and ${ }^{*} P<0.05$ versus respective controls. 


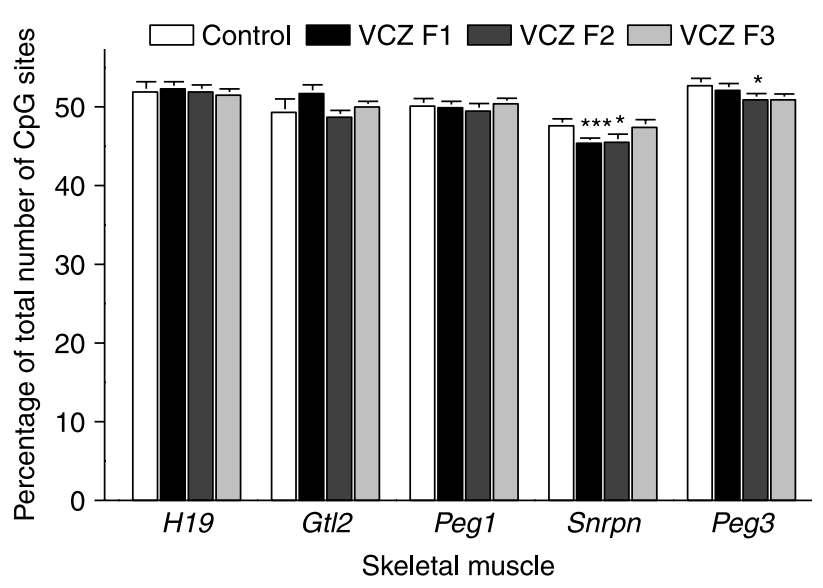

Figure 4 Methylation status of the paternally methylated $\mathrm{H} 19$ and $\mathrm{Gt} / 2$ genes and of the maternally methylated Peg1, Snrpn, and Peg3 genes in the skeletal muscle of the F1-F3 offspring of control and vinclozolinadministered female mice. The results are the means \pm s.E.M. of $8-15$ mice. They represent the number of methylated $\mathrm{CpG}$ sites and are expressed as the percentage of the total number of $\mathrm{CpG}$ sites. ${ }^{* * *} P<0.001$ and ${ }^{*} P<0.025$ versus respective controls.

F1, F2, and F3 offspring of pregnant VCZ-administered female mice. As shown in Fig. 5, the mean sperm concentrations of the VCZ female offspring were only $56 \%$ in the $\mathrm{F} 1,90 \%$ in the $\mathrm{F} 2$, and $100 \%$ in the $\mathrm{F} 3$ of that of the controls.

\section{Discussion}

The current study is the first to address the possible effects of VCZ administration to pregnant female mice on canonical imprinted genes in different tissues, including the male gametes, over three generations.

The pyrosequencing technique was chosen and used throughout this study since it was previously shown to yield results similar to those obtained by the subcloning-sequencing technique (Stouder et al. 2008).

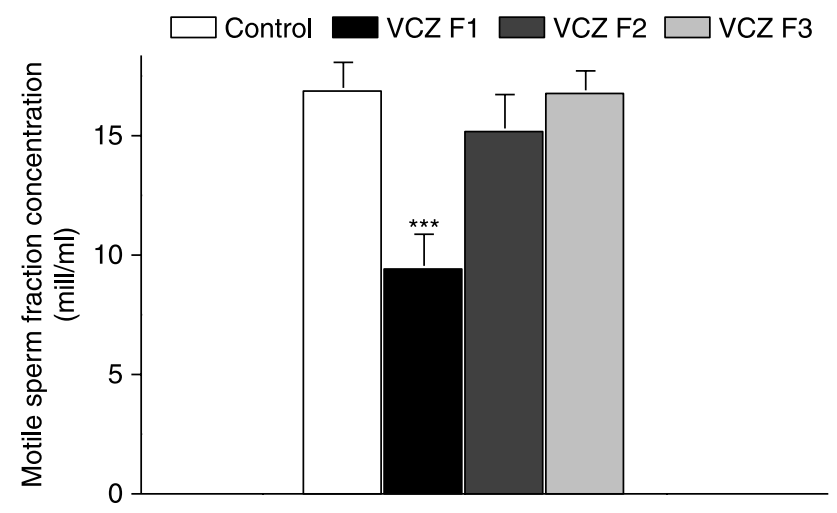

Figure 5 Motile sperm concentration, in millions of spermatozoa per milliliter of sperm, in the F1, F2, and F3 offspring of control and vinclozolin-administered female mice. The results are the means \pm S.E.M. of $5-8$ mice. ${ }^{* * *} P<0.005$.
VCZ was shown to affect the imprinting status of the two paternally (H19 and Gt/2) and three maternally (Peg1, Snrpn, and Peg3) imprinted genes in the sperm of the offspring. These data corroborate the finding by Anway et al. (2005) who showed that VCZ administration to pregnant female mice alters the methylation patterns on 'imprinted-like gene/DNA sequences' in the sperm of the offspring (Anway et al. 2005) and extend it to the well-characterized imprinted genes. Our outcross experiment, showing no phenotype in the sperm of the F2 offspring, confirmed the results of Anway et al. (2006), which suggested that the male germline is responsible for the transmission of the phenotype. In a previous study conducted on the sperm of superovulated female offspring, we described methylation disturbances that affected both paternally and maternally methylated genes in $75 \%$ or more of the F1 offspring (Stouder et al. 2008). The effects of VCZ, described in this study, are more marked and affect $100 \%$ of the F1 offspring.

It is generally admitted that due to the mechanisms of erasure and reprogramming, abnormal epigenetic states are not transmitted to the next generation. However, it has been shown that in some occasions, such as VCZ administration or superovulation in pregnant female mice, epigenetic mark defects can survive transgenerational reprogramming (Anway et al. 2005, Stouder et al. 2008). The possible mechanism of this resistance to reprogramming is not known (Youngson \& Whitelaw 2008). One hypothesis is that it is due to modified expressions of genes coding for proteins involved in the epigenetic reprogramming. In this study, the imprinting defects induced by VCZ in the sperm of the offspring were transgenerational. The methylation, however, showed normal pattern in F3, for three of five imprinted genes. The latter observation suggests that the resistance to reprogramming is reversible and little by little eroded by the successive reprogramming periods occurring during each gestation.

Another finding of this study is that the imprinting errors observed in the sperm of VCZ-administered female mice might have biological consequences as suggested by a decrease in sperm count in the F1 offspring. However, in the F2 and F3 offspring, the sperm count was normalized. There is therefore a discrepancy between the effects of VCZ on the methylation status of sperm imprinted genes which persist in the F2 and F3 offspring and the effect of VCZ on the sperm count, which has already disappeared in the F2 offspring. The lack of correlation between epigenetic and biological changes does not necessarily mean that the decrease in the sperm count observed in the F1 offspring is not related to the changes in the methylation status of sperm imprinted genes. It would be interesting to study whether qualitative parameters of the sperm morphology are or not transgenerationally affected by VCZ.

The methylation errors described in this study have the same characteristics as those reported previously in the offspring of superovulated mothers (Stouder et al. 2008): 
they affect only a fraction of the total number of $\mathrm{CpG}$ sites, but they are highly significant and evenly distributed among the offspring.

Kobayashi et al. (2007) described aberrant methylation patterns of paternally and maternally imprinted genes, and Marques et al. (2010) described a hypomethylation of the paternally imprinted gene $\mathrm{H} 19$ and of its CTFC-binding site 6 in the sperm of patients with abnormal spermatogenesis. In both papers, aberrant methylations as low as $8-10 \%$ of the total number of CpGs were considered in the association studies. Therefore, it is quite possible that small changes in the methylation pattern of imprinted genes, such as those described in this study, might have biological consequences.

In the somatic cells, two different types of changes are observed depending on whether F1 or F2/F3 generations are considered.

In the F1 offspring, the methylation pattern of Snrpn was altered in all somatic cells studied, i.e. those of the tail, liver, and muscle. The occurrence of this alteration in F1 offspring shows that it is not mediated by the germline. It might be the result of a direct toxic effect of VCZ on somatic cells. The reason why this effect targets Snrpn is unknown. The direct alteration of somatic cells is not transmitted to the next generations in the tail and in the liver.

In the F2 offspring, a prominent alteration in the methylation pattern of somatic cells is that occurring in Peg3, which is observed in the tail, liver, and skeletal muscle. In the F3 offspring, interestingly, this alteration is still observed in the tail and liver. The reason why this effect targets Peg3 is unknown. The latter gene encodes a zinc-finger protein that is ubiquitously expressed, although at the highest mRNA levels in the placenta, uterus, ovary, testis, and brain (Hiby et al. 2001). The high conservation of PEG3 protein between mice and humans suggests that it is critical for cellular function. $\mathrm{Peg}^{+/-}$mice demonstrated impaired maternal behavior (Li et al. 1999), impaired olfactory processing (Swaney et al. 2007), a lower growth rate, a delayed puberty, and a tendency to become obese (Curley et al. 2005). The deficits observed in $P$ eg $3^{+/-}$mice might be explained by an increased postnatal apoptosis in the neuroanatomical structures that support reproductive behavior and metabolic homoeostasis (Broad et al. 2009). Therefore, prenatal exposure to VCZ might affect, in addition to sperm concentration, reproductive behavior and energy balance in the offspring. The effects observed in somatic cells of the F2 and F3 offspring are probably transmitted through the germline. We therefore deal with a phenomenon that mimics a DNA mutation, with the important difference, however, being that it seems to be reversible.

Environmental factors affect the methylation pattern of not only imprinted genes but also a number of other target genes throughout the genome (Szyf et al. 2007).
Therefore, our observations, on a selection of strategic sites, might represent only a small fraction of the genome-wide effects of VCZ.

This study shows that VCZ administration to pregnant female mice induces alterations in the imprinted status of five canonical paternally and maternally imprinted genes in the sperm of the offspring which are transgenerational but disappear gradually from F1 to F3. These effects are paralleled by a decrease in the sperm concentration observed only in F1. Therefore, it proposes a mechanism for the previously observed effects of VCZ on the rodent reproductive system (Shimamura et al. 2002, Anway et al. 2005, 2006, 2008, Buckley et al. 2006, Elzeinova et al. 2008). By analogy, we can postulate that the reported effects of EDCs on human male spermatogenesis and fertility rates (Roeleveld \& Bretveld 2008) might involve imprinting alterations in the sperm.

\section{Materials and Methods}

\section{Mice}

Normal FVB/N mice were purchased from Charles River (Arbresle, France). Two-month-old female mice were naturally mated with male mice of the same age. Females with a copulation plug the next morning were separated into two groups. The dose of VCZ reported to affect the male offspring reproductive system when administered to pregnant female rats is $100 \mathrm{mg} / \mathrm{kg}$ per day (Anway et al. 2005, 2006, 2008). Since the mice might be more sensitive to VCZ than the rats and to minimize the possible nonspecific toxic effects of the drug, a dose of $50 \mathrm{mg} / \mathrm{kg}$ per day was chosen. VCZ (Sigma-Aldrich) was suspended in corn oil and administered i.p. to the first group of pregnant mice in doses of $50 \mathrm{mg} / \mathrm{kg}$ per day from days 10 to 18 of pregnancy. The second group consisted of control mice that were injected with only corn oil from days 10 to 18 of pregnancy. To study the possible transgenerational effects, F1 males were analyzed or subsequently crossed with $\mathrm{FVB} / \mathrm{N}$ females in order to obtain F2 males. Both F1 and F2 offspring were used at the age of 2 months. An outcross of a VCZ generation F1 female with a control male was also performed. Animal protocols used in these studies were approved by the Commission d'Ethique de I'Expérimentation Animale of the University of Geneva Medical School and by the Geneva Veterinarian Office.

\section{Sperm collection}

The mouse vas deferens and epididymis were dissected out, placed into a Petri dish, scored with a razor blade in a droplet of PBS into small pieces, and left for 30 min to allow sperm to diffuse into the medium. The latter was then transferred into a microcentrifuge tube, and the fragments were allowed to sediment for $30 \mathrm{~min}$ at $37^{\circ} \mathrm{C}$. The supernatant was carefully transferred to another tube, and this procedure was repeated three times. The final supernatant containing the motile sperm fraction was carefully removed and centrifuged at $6000 \mathrm{~g}$ for $10 \mathrm{~min}$ to pellet the sperm. 


\section{Sperm count}

In some experiments, an aliquot of the supernatant's motile sperm fraction was used for sperm counting using a hemocytometer. The corresponding sperm concentration was calculated using the formula: cells per $\mathrm{ml}=$ the average count per square $\times$ dilution factor $\times 10^{4}$ (counts ten squares).

\section{Tissue collection}

Fragments of $5 \times 5 \mathrm{~mm}$ size of tail, liver, and tibialis anterior muscle were collected, cut into small pieces in a Petri dish, and transferred into DNA-extraction buffer.

\section{DNA isolation}

Motile sperm fraction DNA was extracted using the QIAamp DNA microkit (Qiagen). Total genomic DNA was extracted from tail, liver, and tibialis anterior muscle tissues by incubation for $3 \mathrm{~h}$ at $55{ }^{\circ} \mathrm{C}$ in DNA-extraction buffer $(50 \mathrm{mM}$ Tris- $\mathrm{HCl}, \mathrm{pH} 8,100 \mathrm{mM}$ EDTA, and 0.5\% SDS) followed by phenol-chloroform-isoamyl alcohol extractions and ethanol precipitation.

\section{Bisulfite treatment}

Using the EZ Methylation Gold Kit (Zymo Research, Orange, CA, USA), the extracted DNA was treated with sodium bisulfite in order to convert unmethylated cytosine residues to uracil. The converted DNA was eluted in $10 \mu \mathrm{l}$ TE buffer $(10 \mathrm{mM}$ Tris- $\mathrm{HCl}$ and $0.1 \mathrm{mM}$ EDTA, $\mathrm{pH}$ 7.5). Two microliters of the post-bisulfite-treated DNA were used for subsequent PCR amplification.

\section{PCR amplification of bisulfite-treated DNAs for subsequent pyrosequencing}

The PCR amplifications aimed at pyrosequencing were performed starting from 100 to $140 \mathrm{ng}$ of bisulfite-treated sperm, tail, liver, and skeletal muscle DNAs. The PCR conditions were the same for all the genes tested, i.e. $94{ }^{\circ} \mathrm{C}$ for $15 \mathrm{~min}$, followed by 40 cycles of $94{ }^{\circ} \mathrm{C}, 30 \mathrm{~s} ; 55^{\circ} \mathrm{C}, 30 \mathrm{~s}$; $72{ }^{\circ} \mathrm{C}, 30 \mathrm{~s}$; and by a $72{ }^{\circ} \mathrm{C} 10$-min final extension step.

The characteristics of the amplicons and the oligonucleotides chosen are presented in Table 1.

A diagram showing the locations of the DMDs, within the corresponding genes, and of the DNA fragments of the five genes amplified by PCR has been presented in a previous study (Stouder et al. 2008).

All reactions were performed with a PCR mixture (total volume $25 \mu \mathrm{l}$ ) containing oligonucleotides at $0.5 \mathrm{mM}$ concentration and $12.5 \mu \mathrm{l}$ HotStarTaq Master Mix (Qiagen).

The biotinylated PCR products were purified using streptavidin-sepharose beads (Amersham) and were sequenced using the PSQ 96 Gold reagent kit (Biotage AB, Uppsala, Sweden) with the following primers:

H19: 5'-GTGTAAAGATTAGGGTTGT-3', Gt/2: 5'-GTTATGGATTGGTGTTAAG-3', Peg 1: 5' -TCAATATCAACTAAATAATC-3', Snrpn: 5'-GAATTGGAGTTGTGTGG-3', and Peg3: 5'-AATTGATAAGGTTGTAGATT-3'.
Table 1 Amplicon and oligonucleotide characteristic

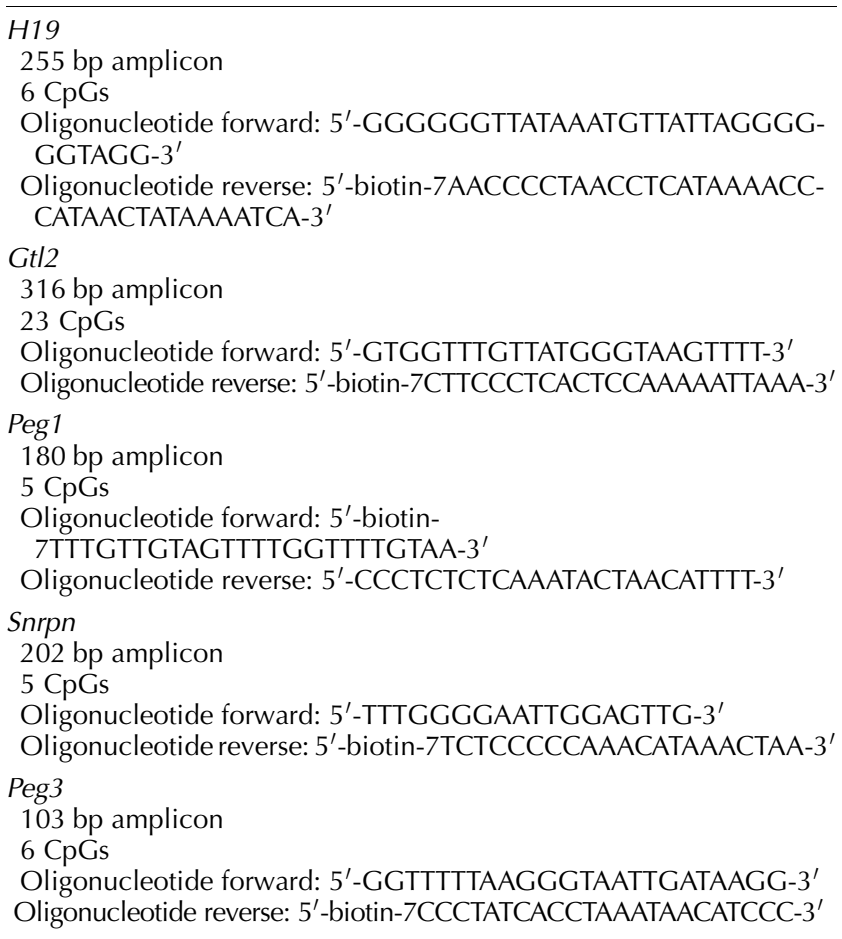

The degree of methylation at each CpG site was determined using Pyro Q-CpG Software (Biotage AB). All samples were analyzed in duplicate.

In a previous study, the results obtained were doublechecked by comparing the techniques used above, i.e. DNA bisulfite treatment and PCR amplification followed directly by pyrosequencing, with PCR amplification followed by amplicon subcloning and sequencing. The results were found to be similar.

\section{Statistical analysis}

Significances were evaluated using unpaired Student's $t$-test and set at $P<0.05$.

\section{Declaration of interest}

The authors declare that there is no conflict of interest that could be perceived as prejudicing the impartiality of the research reported.

\section{Funding}

This work was supported by the FNS grant 3100A0-116021, by the Swiss Academy of Medical Sciences, and the Boninchi Foundation.

\section{Acknowledgements}

We are indebted to Manon Cederroth for excellent technical assistance and to Françoise Kuhne for sperm count procedure setting. 


\section{References}

Andersson AM, Jørgensen N, Main KM, Toppari J, Rajpert-De Meyts E, Leffers H, Juul A, Jensen TK \& Skakkebaek NE 2008 Adverse trends in male reproductive health: we may have reached a crucial 'tipping point'. International Journal of Andrology 31 74-80.

Anway MD, Cupp AS, Uzumcu M \& Skinner MK 2005 Epigenetic transgenerational actions of endocrine disruptors and male fertility. Science 308 1466-1469.

Anway MD, Memon MA, Uzumcu M \& Skinner MK 2006 Transgenerational effect of the endocrine disruptor vinclozolin on male spermatogenesis. Journal of Andrology 27 868-879.

Anway MD, Rekow SS \& Skinner MK 2008 Comparative anti-androgenic actions of vinclozolin and flutamide on transgenerational adult onset disease and spermatogenesis. Reproductive Toxicology 26 100-106.

Broad KD, Curley JP \& Keverne EB 2009 Increased apoptosis during neonatal brain development underlies the adult behavioral deficits seen in mice lacking a functional paternally expressed gene 3 (Peg3). Developmental Neurobiology 69 314-325.

Buckley J, Willingham E, Agras K \& Baskin LS 2006 Embryonic exposure to the fungicide vinclozolin causes virilization of females and alteration of progesterone receptor expression in vivo: an experimental study in mice. Environmental Health 214.

Curley JP, Pinnock SB, Dickson SL, Thresher R, Miyoshi N, Surani MA \& Keverne E 2005 Increased body fat in mice with a targeted mutation of the paternally expressed imprinted gene Peg3. FASEB Journal 19 1302-1304.

Diamanti-Kandarakis E, Bourguignon JP, Giudice LC, Hauser R, Prins GS, Soto AM, Zoeller RT \& Gore AC 2009 Endocrine-disrupting chemicals: an Endocrine Society scientific statement. Endocrine Reviews 30 293-342.

Elzeinova F, Novakova V, Buckiova D, Kubatova A \& Peknicova J 2008 Effect of low dose of vinclozolin on reproductive tract development and sperm parameters in CD1 outbred mice. Reproductive Toxicology 26 231-238.

Hiby SE, Lough M, Keverne EB, Surani MA, Loke YW \& King A 2001 Paternal monoallelic expression of PEG3 in the human placenta. Human Molecular Genetics 10 1093-1100.

Inawaka K, Kawabe M, Takahashi S, Doi Y, Tomigahara Y, Tarui H, Abe J, Kawamura S \& Shirai T 2009 Maternal exposure to anti-androgenic compounds, vinclozolin, flutamide and procymidone, has no effects on spermatogenesis and DNA methylation in male rats of subsequent generations. Toxicology and Applied Pharmacology 237 178-187.

Kaneda M, Okano M, Hata K, Sado T, Tsujimoto N, Li E \& Sasaki H 2004 Essential role for de novo DNA methyltransferase Dnmt3a in paternal and maternal imprinting. Nature 429 900-903.

Kaneko-Ishino T, Kuroiwa Y, Miyoshi N, Kohda T, Suzuki R, Yokoyama M, Viville S, Barton SC, Ishino F \& Surani MA 1995 Peg1/Mest imprinted gene on chromosome 6 identified by cDNA subtraction hybridization. Nature Genetics 11 52-59.

Kobayashi H, Sato A, Otsu E, Hiura H, Tomatsu C, Utsunomiya T, Sasaki H, Yaegashi N \& Arima T 2007 Aberrant DNA methylation of imprinted loci in sperm from oligospermic patients. Human Molecular Genetics 16 $2542-2551$.
Kuroiwa Y, Kaneko-Ishino T, Kagitani F, Kohda T, Li LL, Tada M, Suzuki R, Yokoyama M, Shiroishi T, Wakana S et al. 1996 Peg3 imprinted gene on proximal chromosome 7 encodes for a zinc finger protein. Nature Genetics 12 186-190.

Lee J, Inoue K, Ono R, Ogonuki N, Kohda T, Kaneko-Ishino T, Ogura A \& Ishino F 2002 Erasing genomic imprinting memory in mouse clone embryos produced from day 11.5 primordial germ cells. Development 129 1807-1817.

Li E, Beard C \& Jaenisch R 1993 Role for DNA methylation in genomic imprinting. Nature 366 362-365.

Li L, Keverne EB, Aparicio SA, Ishino F, Barton SC \& Surani MA 1999 Regulation of maternal behavior and offspring growth by paternally expressed Peg3. Science 284 330-333.

Li JY, Lees-Murdock DJ, Xu GL \& Walsh CP 2004 Timing of establishment of paternal methylation imprints in the mouse. Genomics 84 952-960.

Marques CJ, Francisco T, Sousa S, Carvalho F, Barros A \& Sousa M 2010 Methylation defects of imprinted genes in human testicular spermatozoa. Fertility and Sterility [in press]. DOI:10.1016/j.fertnstert.2009.02.051.

Roeleveld N \& Bretveld R 2008 The impact of pesticides on male fertility. Current Opinion in Obstetrics and Gynecology 20 229-233.

Schneider S, Kaufmann W, Buesen R \& van Ravenzwaay B 2008 Vinclozolin - the lack of a transgenerational effect after oral maternal exposure during organogenesis. Reproductive Toxicology 25 352-360.

Shemer R, Birger Y, Riggs AD \& Razin A 1997 Structure of the imprinted mouse Snrpn gene and establishment of its parental-specific methylation pattern. PNAS 94 10267-10272.

Shimamura M, Kodaira K, Kenichi H, Ishimoto $Y$, Tamura H \& Iguchi T 2002 Comparison of antiandrogenic activities of vinclozolin and D,Lcamphorquinone in androgen receptor gene transcription assay in vitro and mouse in utero exposure assay in vivo. Toxicology 174 97-107.

Smilinich NJ, Day CD, Fitzpatrick GV, Caldwell GM, Lossie AC, Cooper PR, Smallwood AC, Joyce JA, Schofield PN, Reik W et al. 1999 A maternally methylated CpG island in KvLQT1 is associated with an antisense paternal transcript and loss of imprinting in BeckwithWiedemann syndrome. PNAS 96 8064-8069.

Stouder C, Deutsch S \& Paoloni-Giacobino A 2008 Superovulation in mice alters the methylation pattern of imprinted genes in the sperm of the offspring. Reproductive Toxicology 25 352-360.

Swaney WT, Curley JP, Champagne FA \& Keverne EB 2007 Genomic imprinting mediates sexual experience-dependent olfactory learning in male mice. PNAS 104 6084-6089.

Szyf M, Weaver I \& Meaney M 2007 Maternal care, the epigenome and phenotypic differences in behavior. Reproductive Toxicology 24 9-19.

Tremblay KD, Duran KL \& Bartolomei MS 1997 A 5' 2-kilobase-pair region of the imprinted mouse $\mathrm{H} 19$ gene exhibits exclusive paternal methylation throughout development. Molecular and Cellular Biology 17 4322-4329.

Youngson NA \& Whitelaw E 2008 Transgenerational epigenetic effects. Annual Review of Genomics and Human Genetics 9 233-257.

Received 11 August 2009

First decision 22 September 2009

Revised manuscript received 8 October 2009

Accepted 3 November 2009 